\title{
Intracranial Brain Volume (ICV) Measurement in Epileptic Male Patient: A 3D CT Study
}

\author{
Lubna Shirin,,${ }^{1,2}$ Nor Farid Mohammed Noor, ${ }^{1}$ Tahamina Begum, ${ }^{3}$ Hadif Zaidin Samsudin, ${ }^{4}$ Rehana Basri, ${ }^{5}$ \\ Johari Yap Abdullah, ${ }^{6}$ Mohammed Shahjahan Kabir, ${ }^{7}$ Mohammad Khursheed Alam ${ }^{8}$
}

\begin{abstract}
Objective: Intracranial volume (ICV) is one of the reliable indicators of neurodegenerative disease and premature brain size. Epilepsy is considered a neurological disorder. We aimed to measure ICV in epileptic male samples to identify the relation of ICV and epilepsy control status for their better treatment purpose.

Methods: This retrospective study was done using CT images of age-matched control and epileptic male samples. All samples were collected from the archive of the Department of Radiology, Universiti Sains Malaysia (USM) from the 2010-2017 period. A total of 34 male samples were used for this study in two groups, control $(n=17)$ and epileptic $(n=17)$ groups. Control males were those who came for the CT scan and no disease was found. And epileptic male patients were those who came for routine checkups due to epilepsy. MITK 3 M3 software was used for the ICV measurement. 2D CT images were converted to 3D CT images to measure intracranial brain volume (ICV) in each group. Two reviewers measured ICV and a reliability test was done between reviewers.

Results: According to first reviewer, there is no significant $(p=0.455)$ difference between control, ( 1287.82 $(15 I .79) \mathrm{mm}$ ) and epileptic (I283.28 (65.48) $\mathrm{mm}$ ) male groups. Results of second reviewer also showed no significant difference $(p=0.400)$ between control $($ I $299.58($ I44.8I)) $\mathrm{mm}$ and epileptic (I283.88 (76.08)) groups. Average measurements also did not reveal any significant difference between groups, control is I 293.7 ( I 44.8I) $\mathrm{mm}$ and the epilepsy group is 1283.58 (69.90) $\mathrm{mm}(p=0.1$ I 4). Reliability test results revealed an acceptable internal consistency level in control $(97 \%, p<0.00 I)$ and epileptic $(77 \%, p=0.003)$ groups.

Conclusion: We concluded that epilepsy does not affect ICV in the male population. Further study is recommended to seek other indicators which might be affected by epilepsy in the male population.

Keywords: Epilepsy, 3D CT, MITK 3M3

(c) (i) (2) (c) ${ }_{\mathrm{BY}} \mathrm{NC}$ SA

DOI: https://doi.org/l0.3329/jom.v22i2.56697 Copyright: (C) 202I Shirin L. This is an open access article published under the Creative Commons Attribution-NonCommercial-NoDerivatives 4.0 International License, which permits use, distribution and reproduction in any medium, provided the original work is properly cited, is not changed in any way and it is not used for commercial purposes.
\end{abstract}

Received: 20 October, 2020;

Accepted: 13 January 202I

1. Department of Anatomy, School of Dental Sciences, Universiti Sains Malaysia, 16150 Kubang Kerian, Kota Bharu, Kelantan, Malaysia.

2. Department of Anatomy, Faculty of Medicine, QUEST International University Perak, Ipoh, Perak, Malaysia.

3. Department of Neurosciences, School of Medical Sciences, Universiti Sains Malaysia, 16150 Kubang Kerian, Kota Bharu, Kelantan, Malaysia.

4. Department of Radiology, School of Medical Sciences, Universiti Sains Malaysia, 16150 Kubang Kerian, Kota Bharu, Kelantan, Malaysia.

5. College of Medicine, Jouf University, Kingdom of Saudi Arabia.

6. Craniofacial Medical Imaging Research group, School of Dental Sciences, Universiti Sains Malaysia, 16150 Kubang Kerian, Kota Bharu, Kelantan, Malaysia.

7. Department of Medicine, Faculty of Medicine, QUEST International University Perak, Ipoh, Perak, Malaysia.

8. Orthodontic Unit, College of Dentistry, Jouf University, Sakaka, Kingdom of Saudi Arabia

Corresponding author: Dr. Nor Farid Mohammed Noor 1\&2) Department of Anatomy, School of Dental Sciences, Universiti Sains Malaysia, 16150 Kubang Kerian, Kota Bharu, Kelantan, 95alaysia. Email: drfarid@usm.my Phone: HP:+60177161929

\section{Introduction:}

Intracranial volume (ICV) is well-defined as the volume within the cranial cavities including the brain, meninges, and cerebrospinal fluid ${ }^{1}$. The volume rises from birth throughout childhood. The maximum growth is completed in the first five years $^{2}$. The ICV reaches its final size at the age of 16-20 years and it was supposed that it does not change its size thereafter 3 .At about 20 years of age, the brain volume begins to decrease, however, it is assumed that ICV is directly correlated with age, sex, social class and, race. And the measurement of ICV is an important indicator of brain development ${ }^{4}$. Generalized cerebral volume loss refers to a loss of brain cells affecting the whole brain. Cerebral atrophy might happen in dementia, seizures, aphasia, etc. Patients with epilepsy might have structural abnormalities, including cortical thickness, volume reduction, and reduced hippocampal volume ${ }^{5,6}$. Diffuse cortical thinning is found in both ipsilateral, contralateral temporal, and extratemporal regions in temporal lobe epilepsy (TLE) ${ }^{7}$. Hence, this is important to know the measurement of ICV among epilepsy patients. 
There are various ways to measure ICV. Length of the ventricular size and the subarachnoid space has been performed by a two-dimensional method using single CT scan slices by packing method using filling materials such as millet and mustard seeds ${ }^{8}$. Recently, magnetic resonance imaging (MRI) and CT images have made conceivable accurate measurements of $\mathrm{ICV}^{9,10}$. It helps to give us quantifiable information from slices taken through different parts of the living brain. Bonoventura Cavalieri an Italian mathematician made significant developments in the mathematics of numerical integration and initially consider measurement of volume through the analysis of sections of three-dimensional solid objects ${ }^{11,12}$ on $\mathrm{CT}$ images of Australian normal populations by using an in-house Persona 3D software package. Persona software maintains the contours of bone in each slice and saves them into separate files. The ICV is calculated by summing the cross-sectional areas that intersect the region of interest and multiplying by slice separation which they denoted as Cavalieri estimator. Likewise, the same method was also used to measure ICV of non-syndromic sagittal synostosis ${ }^{13}$ and Apert syndrome patients ${ }^{14}$. There is still limited information about the measurement of ICV in epilepsy patients, meaning that ICV measurement might or might not be changed during epilepsy. Taking this question, we aimed to investigate ICV measurement among epilepsy male samples that compare with the control male samples using CT images, retrospectively. In this present study, we used only male samples as there are differences in ICV measurement between male and female ${ }^{4}$. Combining male and female populations might explore the confusing results due to their normal ICV difference, Hence, in this study, we focused only male population.

In a retrospective study, the reliability test can be done to check the internal consistency level among reviewers. The internal consistency level is measured by Cronbach's alpha values ${ }^{15}$. More closer the values to 1 , indicates the greater reliability. In exploratory research, the acceptable value for Cronbach's alpha is 0.6 but usually, the acceptable value is 0.7. Cronbach's alpha scale is like this: very high $(>0.90)$, high (0.75-0.90), moderate (0.60-0.75), low (0.30-0.60) and very low $(<0.30)^{16,17}$. In this present retrospective study, ICV was measured by two reviewers between control and epileptic male samples with a 3D CT scan using MITK 3M3 software.

\section{Materials \& Methods:}

Ethical permission and sample collection

Before collecting sample data, we received ethical permission from the ethical committee of Universiti Sains Malaysia (USM) (USM/JePEM/17030155). In this retrospective study, a total of 34 male samples were used in two groups; control $(n=17)$ and epileptic $(n=17)$ groups. Power and sample size (PS) software was used to calculate the sample number between groups. The mean difference $(\delta)$ of ICV measurement between groups and standard deviation difference $(\sigma)$ were 0.02 and 0.02 , respectively ${ }^{2} . \beta$ and $\alpha$ values were 0.8 and 0.05 , respectively. CT images were collected from the archive of the radiology department of USM. As these are the administrative data, we collected permission from the Hospital director. All the samples were males and samples in both groups were age-matched. Inclusion criteria were set for both groups as 1) gender male 2) age range was 30-60 years old 3) no major diseases like hypertension, diabetes, asthma, psychological problem, etc because these diseases might affect normal ICV measurement. ${ }^{18,19,21}$ Moreover, for the control group, control samples were collected from those who came for a CT scan, and no disease was diagnosed. For epileptic groups, epileptic samples had a history of epilepsy and antiepileptic drug (AED) history for more than five years.

\section{The procedure of ICV measurement}

CT images were retrieved from the PACS Server to a Dell Precision T7500 workstation in Digital Imaging and Communications in Medicine (DICOM) format. MITK 3M3 software was used to measure ICV in CT images with the Cavalieri method. First, we calculated the first axial slice at the vertex where the meninges appeared until the last axial slice at the foramen magnum. The process was repeated in each axial slice, from the vertex to the foramen magnum, manually we inserted the CT scan slice ${ }^{10}$ and it came automatically during volume measurement until the entire regions of the intracranial area were segmented automated technique. The 1-in-10 strategies were used to obtain an estimate of the ICV based on the Cavalieri method, from the vertex to the foramen magnum. The sum of the areas was multiplied by the slice thickness, which was $10 \mathrm{~mm}$ per the sampling strategy. For the 1-in-10 strategies, the number of slices was processed by reduced considerably from about 200 slices to about 20 and 40 slices. Once the Cavalieri method was applied to estimate the ICV, the 2D CT images were converted to $3 \mathrm{D}$ CT images with extracted quantifiable information. Final ICV measurements were done by contour interpolation in 3D CT images (Figure 1). 

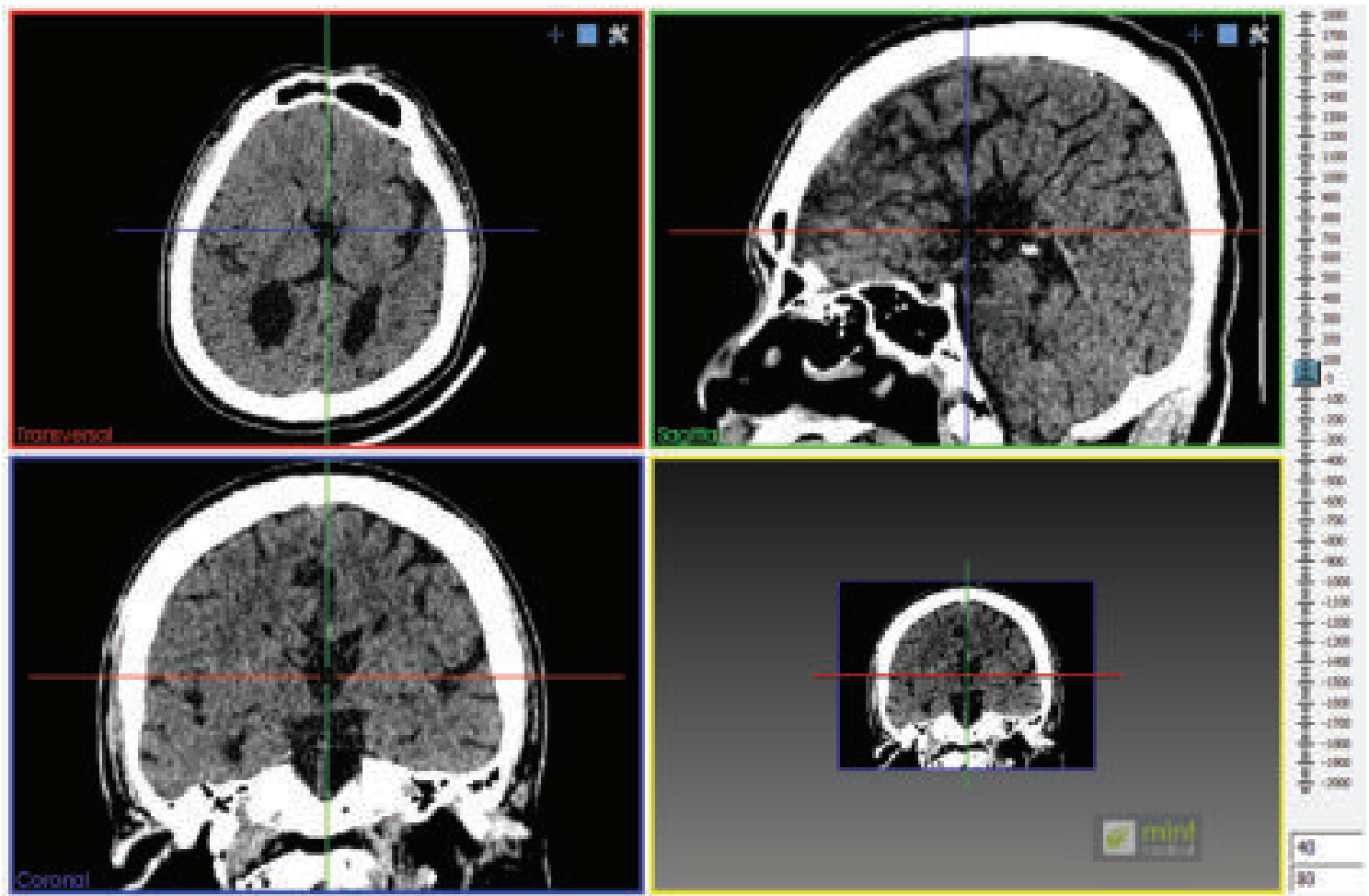

Figure 1: The four-window view showing measurement of intracranial brain volume (ICV) using MITK $3 M 3$ software the crosshair pointing at the same location where all lines cross each other. Top left = axial view; top right = sagittal view; bottom left coronal view; bottom right $=3 D$ reconstruction view.

\section{Statistical analysis}

Statistical Package for the Social Sciences (SPSS) 24 software (Chicago, IL, USA), was used to find the significant difference between groups (Mann-Whitney U test), and a reliability test was used to find internal consistency level between reviewers. $p$-value was determined as less than 0.05 .

\section{Results:}

Demographic information of both groups showed that the mean (SD) ages were 32.88 (14.74) years and 36.05 (13.91) years in the control and epilepsy groups, respectively (Table I). There is no significant $(p=0.480)$ difference in ages between both groups.

Table-I. Demographic information of control and epileptic males:

\begin{tabular}{lcc}
\hline Groups & Control group & Epileptic group \\
\hline Ages, Mean (SD) in years & $32.88(14.74)$ & $36.05(13.91)$ \\
\hline
\end{tabular}

Intracranial brain volumes (ICV) were measured by two reviewers. To observe significant differences between groups, we performed a non-parametric Mann-Whitney test separately on the ICV measurements of $1^{\text {st }}$ reviewer, $2^{\text {nd }}$ reviewers, and also with their averages to check the significant difference of ICV measurement between groups by two separate reviewers. Moreover, interobserver variation was checked with their average results. Results showed that there was no significant difference in ICV measurement between control and epileptic groups for the 1 st $(p=0.455)$ and 2 nd reviewers $(p=0.400)$. The median (IQR) measurements of the 1st reviewer were 1287.82 (151.79) $\mathrm{ml}$ in the control group and $1283.28(65.48) \mathrm{ml}$ in the epileptic group. The median (IQR) measurements of the 2nd reviewer were 1299.58 (141.90) $\mathrm{ml}$ in the control group and $1283.88(76.08) \mathrm{ml}$ in the epileptic group. The average median (IQR) measurements for the control group were $1293.7(144.81) \mathrm{ml}$ and in the epileptic group was 1283.58 (69.90) $\mathrm{ml}$ and no significant $(p=0.114$ ) difference between groups (Table II).

A reliability test was done between reviewers. We found a very high level (97\%) internal consistency level in the control group between reviewers and a high level (77\%) internal consistency level in epileptic groups. Cronbach's alpha scores were 0.979 ( $p \hat{A} 0.001$ ) and 0.773 (0.003) in control and epileptic groups, respectively. 
Table-II. ICV median (IQR) measurements of two reviewers and an average of them, were shown between two groups

\begin{tabular}{lccc}
\hline ICV measurements & Control group, Median (IQR) in ml & Epileptic group,Median (IQR) in ml & $p$-value \\
\hline $1^{\text {st }}$ reviewer & $1287.82(151.79)$ & $1283.28(65.48)$ & 0.455 \\
$2^{\text {nd }}$ reviewer & $1299.58(141.90)$ & $1283.88(76.08)$ & 0.400 \\
Average & $1293.7(144.81)$ & $1283.58(69.90)$ & 0.114 \\
\hline
\end{tabular}

\section{Discussion:}

In this retrospective study, we aimed to investigate the ICV measurement between the control and epileptic male population. ICVs were measured by two reviewers. There was no significant difference in ICV measurements between the control and epileptic male groups in the two reviewers. Their average also did not show any significant difference. The internal consistency level was acceptable between reviewers.

A magnetic resonance imagining (MRI) study in male Alzheimer patients showed lower ICV measurement comparing with the control male ${ }^{18}$. All the male patients of bipolar disorder, schizoaffective, and schizophrenia, showed lower ICV measurement with control male ${ }^{19}$. Craniosynostosis male patients showed lower ICV measurement in CT images ${ }^{20}$. Higher ICV measurement was found in male Idiopathic normal-pressure hydrocephalus (NPH) ${ }^{21}$. There is no study yet in epileptic male patients to measure ICV in 3D CT images. In our present retrospective study, we did not found any significant difference in epileptic males comparing with the control male group, which contradicted results with the other studies ${ }^{18-21}$. Our result explores that epilepsy disease might have no effect on ICV among male patients, mainly when all the epileptic patients were taking antiepileptic drugs (AEDs) for more than 5 years. It might be also no effect of AEDs on ICV in male epileptic patients.

Some limitations were in this present study, 1) a small sample size. We need a large sample to get reliable information 2) to use images from different technologies like MRI and compare them with our result.

\section{Conclusion:}

This retrospective study was done to investigate the measurement of ICV in male epileptic samples in 3D CT images. No significant differences were found between groups that indicated that epilepsy disease has no effect on ICV in male epileptic patients.

\section{Acknowledgement:}

The present study was approved by the Human ethical permission by the ethical committee of HUSM was received (RefNo: USM/JePEM/17030155)

\section{Conflict of interest: None}

\section{References:}

1. Whitwell J L, Crum W R, Watt H C, and Fox N C. Normalization of cerebral volumes by use of intracranial volume: Implications for longitudinal quantitative MR imaging. Am J Neuroradiol 2001; 22 (8): 1483-1489.

2. Sgouros S Goldin, J H Hockley, A D Wake, M J, and Natarajan K. Intracranial volume change in childhood. J Neurosurg 1999; 91 (4): 610-616.

3. Wolf H, Kruggel F, Hensel A, Wahlund L O, Arendt T, and Gertz H J.. The relationship between head size and intracranial volume in elderly subjects. Brain Res 2003; 973(1): $74-80$.

4. Rushton J P, and Ankney, C. D. Brain size and cognitive ability: Correlations with age, sex, social class, and race. Psychonomic Bull Rev 1996; 3(1): 21-36.

5. Bernhardt BC, Worsley KJ, Besson P, Concha L, Lerch JP, Evans AC, Bernasconi N. Mapping limbic network organization in temporal lobe epilepsy using morphometric correlations: insights on the relation between mesiotemporal connectivity and cortical atrophy. Neuroimage 2008; 42:515524.

6. Bonilha L, Rorden C, Appenzeller S, Coan AC, Cendes F, Li LM. Gray matter atrophy associated with duration of temporal lobe epilepsy. Neuroimage 2006; 32:1070-1079.

7. Lawson JA, Cook MJ, Vogrin S, et al. Clinical, EEG, and quantitative MRI differences in pediatric frontal and temporal lobe epilepsy. Neurology 2002 ; 58:723-29.

8. Manjunath K Y. Estimation of cranial volume: An overview of methodologies. J Anat Soc India 2002b; 51(1): 85-91.

9. Ambarki K, Lindqvist T, Wahlin A, Petterson E, Warntjes M J, Birgander R, Malm J, and Eklund A. Evaluation of automatic measurement of the intracranial volume based on quantitative MR imaging. Am J Neuroradiol 2012;33 (10): 1951-1956.

10. Embong M F, Yaacob R, Abdullah M S, Abdul Karim A H, Ghazali A K, and Jalaluddin S. MR volumetry of hippocampus in normal adult Malay of age 50 years old and above. Malays J Med Sci 2013; 20(4): 24-30. 
11. Roberts N, Puddephat M J, and McNulty V. The benefit of stereology for quantitative radiology. Br J Radiol 2000; 73 (871): 679-697.

12. Abbott A H, Netherway D J, Niemann D B, Clark B, Yamamoto M, Cole J, Hanieh A, Moore M H, and David D J. CT-determined intracranial volume for a normal population. J Craniofac Surg 2000; 11 (3): 211-223.

13. Anderson P J, Netherway D J, McGlaughlin K, and David D J. Intracranial volume measurement of sagittal craniosynostosis. J Clin Neurosci 2007; 14 (5): 455-458.

14. Anderson P J, Netherway D J, Abbott A H, Cox T Roscioli, T and David D J. Analysis of intracranial volume in Apert syndrome genotypes. Pediatr Neurosurg 2004 ; 40(4): 161164.

15. Cortina JM. What Is Coefficient Alpha? An Examination of Theory and Applications. Journal of Applied Psychology 1993; 78(1):98-104.

16. Cunha CM, Almeida Neto OP, Stackfleth R. Main psychometric evaluation methods of measuring instruments reliability. Rev Atenção Saúde. 2016; 14(49): 98-103. doi: 10.13037/rbcs.vol14n49.3671
17. Malacarne MP, Luiz SG, Amaral TR, Siqueira MM. Health service evaluation in Public Health: a survey of research on assessment in Public Health Graduate Programs. Rev Bras Pesqui Saúde. 2017; 18(1):62-7. doi: https://doi.org/ 10.21722/rbps.v18i1.15136

18. Jenkins R, Fox NC, Rassor MA. Harvey JH, Rassor NM. Intracranial volume and Alzheimer Disease. Arch Neurol 2000 ; 57:220-224.

19. Reite M, Reite E, Collins D, Teale P, Rojas CD, Sandberg E. Brain size and brain/intracranial volume ratio in major mental illness. BMC Psychiatry 2010; 10:79. http:// www.biomedcentral.com/1471-244X/10/79.

20. Amanda H Abbott, et al. CT-Determined Intracranial Volume for a Normal Population. Journal of Craniofacial Surgery 2000; 11(3):211-23. DOI: 10.1097/00001665-20001103000002 .

21. William G Bradley, Francis G Safar, Claudia Hurtado, Justin Ord, and John F. Alksne Increased Intracranial Volume: A Clue to the Etiology of Idiopathic Normal-Pressure Hydrocephalus? AJNR Am J Neuroradiol 2004; 25:14791484. 